\title{
Transfert de connaissances issues de la recherche en éducation : situation globale, défis et perspectives
}

\section{The transfer of research knowledge in education: Overview,} challenges and prospects

\section{Transferencia de conocimientos procedentes de la investigación en educación: situación global, desafíos y perspectivas}

\section{Caroline Marion et Nathalie Houlfort}

Volume 18, numéro 2, 2015

URI : https://id.erudit.org/iderudit/1036033ar

DOI : https://doi.org/10.7202/1036033ar

Aller au sommaire du numéro

Éditeur(s)

Faculté d'éducation, Université de Sherbrooke

ISSN

1911-8805 (numérique)

Découvrir la revue

Citer cet article

Marion, C. \& Houlfort, N. (2015). Transfert de connaissances issues de la recherche en éducation : situation globale, défis et perspectives. Nouveaux cahiers de la recherche en éducation, 18(2), 56-89.

https://doi.org/10.7202/1036033ar

\section{Résumé de l'article}

Le transfert des connaissances issues de la recherche s'impose comme un champ d'investigation incontournable dans plusieurs domaines. Tant en santé, en sciences sociales, humaines et appliquées qu'en éducation ou en gestion, des recherches visent à réduire le fossé entre les connaissances produites et ce qui est observé dans la pratique. Découlant d'une recension des écrits publiés entre 2007 et 2013, le présent article propose de situer le transfert des connaissances en éducation au regard de ce qui se passe globalement dans les autres domaines. Il en aborde les distinctions ainsi que les défis et les perspectives. 


\section{Transfert de connaissances issues de la recherche en éducation: situation globale, défis et perspectives}

\section{Caroline Marion \\ Nathalie Houlfort}

Université du Québec à Montréal

\section{Résumé}

Le transfert des connaissances issues de la recherche s'impose comme un champ d'investigation incontournable dans plusieurs domaines. Tant en santé, en sciences sociales, humaines et appliquées qu'en éducation ou en gestion, des recherches visent à réduire le fossé entre les connaissances produites et ce qui est observé dans la pratique. Découlant d'une recension des écrits publiés entre 2007 et 2013, le présent article propose de situer le transfert des connaissances en éducation au regard de ce qui se passe globalement dans les autres domaines. Il en aborde les distinctions ainsi que les défis et les perspectives.

Mots clés: transfert des connaissances, pratique, recherche, formation, éducation

\section{The transfer of research knowledge in education: Overview, challenges and prospects}

\section{Abstract}

The transfer of research knowledge has become a key area of investigation in a number of fields. Whether in the health, social, human and applied sciences, in education, or in management, research continually seeks to narrow the gap between the knowledge that is produced and the realities that are observed in practice. Based on a review of the literature published between 2007 and 2013, this article sets out to situate 
knowledge transfer in education with respect to what is going on globally in other fields. Accordingly, the text addresses distinctions as well as challenges and prospects.

Key words: knowledge transfer, practice, research, training, education

\section{Transferencia de conocimientos procedentes de la investigación en educación: situación global, desafíos y perspectivas}

\section{Resumen}

La transferencia de conocimientos procedentes de la investigación se impone como un campo de investigación ineludible en varias áreas. Tanto en la salud, en ciencias sociales, humanas y aplicadas como en educación o en gestión, las investigaciones tienden a reducir la brecha entre los conocimientos producidos y lo que se observa en la práctica. El presente artículo es el resultado de una revisión bibliográfica publicada entre 2007 y 2013. Tiene por propósito ubicar la transferencia de conocimientos en educación referente a lo que sucede globalmente en las demás áreas. Trata de las distinciones así como de los desafíos y perspectivas que implica esa transferencia.

Palabras clave: transferencia de conocimientos, práctica, investigación, formación, educación

\section{Introduction}

En éducation comme dans d'autres secteurs, le bassin des connaissances issues de la recherche (CIR) est non seulement abondant, mais continue de croître année après année (Albaladejo, Geslin, Magda et Salembier, 2009; Lenoir et Vanhulle, 2006). Ces connaissances ont une valeur sociale. En effet, bon nombre d'entre elles peuvent servir de guides en termes de comportements et de pratiques plus efficaces pour des utilisateurs, qu'il s'agisse de professionnels en exercice ou de décideurs (Becheikh, Ziam, Idrissi, Castonguay et Landry, 2010; Harvey, 2011). Or, pour que ces CIR puissent avoir une portée dans l'action, encore faut-il qu'elles soient transférées. Des données révèlent pourtant que leur transfert est déficitaire. Malgré les efforts entrepris, il y aurait un écart persistant entre les CIR et leur utilisation dans plusieurs domaines dont l'éducation (Abrami, Lysenko, Janosz, Bernard et Dagenais, 2010; Levin, 2010). Par le fait même, 
certains publics bénéficiaires de services se voient privés d'un accès aux pratiques plus efficaces (Crona et Parker, 2011; Dagenais, Janosz et Dutil, 2008; Gervais, Chagnon et Paccioni, 2011). Dans cette optique, il n'est pas étonnant que maintes études aient été produites en vue de parfaire notre compréhension et notre action pour un meilleur transfert des connaissances (TC). Or, avant de poursuivre l'agenda de recherche dans cette direction, il parait nécessaire de faire le point sur l'avancement des connaissances sur le transfert des CIR. Nous proposons de le faire ici pour le domaine de l'éducation.

\section{Problématique}

En éducation, le TC est un problème d'envergure, et cela, malgré une abondance de connaissances (Albaladejo et al., 2009). Uniquement sur le concept de "réussite scolaire», il s'est produit plus de 45000 publications scientifiques et professionnelles ces dix dernières années ${ }^{1}$. Le domaine comporte ainsi un vaste réservoir de CIR touchant à une multitude de thèmes: apprentissage des élèves (Perrenoud, 2008), motivation et persévérance à l'école (Gurtner, Gulfi, Monnard et Schumacher, 2005), mode de gestion des directions (Thibodeau, Dussault, Frenette et Royer, 2011), facteurs familiaux agissant sur la réussite scolaire (Deslandes et Bertrand, 2004), etc. Or, pour que ces connaissances puissent se traduire par des pratiques plus efficaces et une réussite scolaire rehaussée, encore faut-il qu'il y ait transfert. Des études révèlent pourtant que le transfert des CIR dans les milieux scolaires est des plus complexes et difficiles (Cooper, 2013; Dagenais, Lysenko, Abrami, Bernard, Ramdé et Janosz, 2012; Levin, 2010). Certains dénoncent d'ailleurs l'existence d'un écart important entre les CIR et les pratiques observées en milieu scolaire (Brown, 2012; Davidson et Nowicki, 2012; Lemire, Bigras et Eryasa, 2011).

Différentes causes peuvent expliquer ces résultats mitigés: 1) le faible degré d'autonomie des enseignants quant à leur développement professionnel (Butler, Novak Lauscher, Jarvis-Selinger et Beckingham, 2004); 2) le rapport au savoir plutôt pratique que théorique des enseignants, directions et conseillers pédagogiques (Fournier, 2011; Maubant et Roger, 2012); 3) le type de candidats qui choisissent le travail d'enseignant alors que leurs intentions se révèlent en incohérence ou éloignées de la mission enseignante (Deauvieau, 2005; Rioux et Chevrollier, 2009); 4) l'insuffisance de recherches-actions et de recherches collaboratives dans les écoles (Dionne, 2003; Perrenoud, Altet, Lessard et Paquay, 2008); 5) des décideurs mal formés, n'orientant pas toujours correctement les actions posées en matière de TC, privilégiant parfois eux-mêmes des formations courtes au lieu d'investir dans un processus de formation continue (Levin, Cooper, Arjomand et 
Thompson, 2011); 6) des décisions politiques au regard des partis en place de nature à freiner l'innovation en lien avec le TC (Bowen, Martens et al., 2005); et 7) des tensions résultant de la mission universitaire au regard du mandat de recherche et du mandat de nature professionnelle des chercheurs (Dagenais et al., 2008; Ridde, 2009).

Si ces éléments et d'autres sont en cause dans la faible utilisation des CIR en éducation, des facteurs concernent plus particulièrement: 1) le type de CIR produit en éducation et 2) la manière d'opérationnaliser le TC dans les milieux de pratique. Des auteurs décrient ainsi un trop grand nombre de CIR non contextualisées, peu pertinentes pour les réalités et problèmes des intervenants scolaires (Crahay, Wanlin, Issaieva et Laduron, 2010; Hedges, 2010; Lemire et al., 2011). Les stratégies et plans d'action pour favoriser le TC dans les milieux ne prendraient pas suffisamment en compte les besoins des utilisateurs en termes d'accompagnement, de mesures de soutien et de temps octroyé (Abrami et al., 2010; Gagnon, 2012; Landry, Becheikh, Amara, Ziam, Idrissi et Castonguay, 2008). Pour d'autres encore, les approches de TC prisées au plan organisationnel consistent trop souvent en des gestes et initiatives isolés au lieu de démarches systémiques (Levin et al., 2011; Landry et al., 2008). Des recherches conduites en éducation témoignent en outre de l'importance de l'interactivité dans le TC, de la présence d'échanges formels et informels entre producteurs (ou accompagnateurs) et utilisateurs (Becheikh et al., 2010; Campbell, 2011; Cooper, 2013; Sa, Li et Faubert, 2010).

En éducation, l'enjeu principal du TC est la réussite scolaire des apprenants (Lamari, 2012; Levin et al., 2011). Pour les intervenants scolaires, le transfert des CIR (en termes de meilleures réponses, pratiques ou solutions) favorise non seulement l'apprentissage académique, mais aussi le développement optimal des élèves (Chabot, 2007; Ramdé, 2012; Shonkoff et Bales, 2011). Pour les responsables et les gestionnaires en éducation, le TC permet des décisions professionnelles plus judicieuses à moyen et à long terme (Lamari, 2012; Shonkoff et Bales, 2011). Ainsi, que ce soit pour l'amélioration du climat de travail, la planification d'objectifs ou la mise en place de stratégies liées à la réussite, le TC peut jouer un rôle important (Landry et al., 2008). Finalement, considérant les fonds publics investis année après année dans le domaine, il est justifié d'associer le TC en éducation à une reddition de comptes pour les citoyens (Landry, 2010).

En résumé, au cœur du problème attenant au faible transfert des CIR dans les milieux scolaires se trouve la sous-utilisation de pratiques révélées plus efficaces pour la réussite scolaire et la gestion optimale de l'école. Des connaissances en termes de 
pratiques exemplaires existent et sont entreposées dans des lieux (virtuel, sous format papier), pourtant, peu sont transférées jusqu'à leur utilisation par différents intervenants scolaires. Ces $\mathrm{CIR}$ répondent pourtant à des problématiques scolaires d'importance comme la prévention du décrochage scolaire, la réussite des élèves plus à risque, l'engagement des enseignants, etc. Au vu des données précédentes, il nous parait pertinent de faire le point sur la situation globale du transfert des connaissances en éducation.

Nous avons donc réalisé un scoping study, soit une étude de cadrage portant sur des concepts clés, analysés et traités au travers d'un processus itératif en vue de tracer le portrait d'une situation (Arksey et O'Malley, 2005). L'étude a pour objectif de situer le transfert des connaissances en éducation au regard de ce qui se passe globalement dans les autres domaines que sont: les sciences de la santé, les sciences sociales et les sciences de la gestion. Notre projet vise également à identifier les défis et les perspectives du TC en éducation. Les prochaines sections traitent du cadre théorique, de la méthodologie utilisée et des résultats obtenus. Une discussion servant à identifier les lacunes du TC en éducation et des suggestions pour y remédier clôture l'article.

\section{Cadre théorique}

Dans cette section, nous explicitons le concept de "connaissances issues de la recherche (CIR)" ainsi que celui de "transfert des connaissances" au vu des écrits parus dans le domaine de l'éducation. De plus, nous précisons notre choix de recourir au concept de "transfert des connaissances" pour le présent article.

\subsection{Connaissances issues de la recherche (CIR)}

Pour Saussez et Paquay (2004), les connaissances issues de la recherche en éducation sont autant de concepts scientifiques qui s'amalgament aux concepts quotidiens des praticiens, ces derniers provenant d'expériences antérieures et de socialisations diverses. Fruits de la recherche, les connaissances scientifiques s'apparentent à des lignes directrices servant à guider l'action, apportent un éclairage aux situations et à leur contexte. Les auteurs précisent que les concepts quotidiens (savoirs d'expérience) jouent aussi ce rôle et que c'est la confrontation entre ces deux types de concepts qui crée des tensions et des résistances chez les intervenants scolaires. Selon cette position, le rôle des CIR serait de justifier les actions, voire de guider ou d'éclairer les décisions en contexte scolaire, sur le plan pédagogique et didactique notamment. 
Similairement, Brodeur, Deaudelin et Bru (2005) suggèrent que les CIR sont des connaissances qui permettent à des acteurs tels les enseignants, par exemple, d'adapter "leurs pratiques de diagnostic, d'intervention et d'évaluation" (p. 9) de façon à intervenir de façon plus efficace auprès des élèves. Colluci-Cray et Fraser (2008) présentent les CIR comme des savoirs servant à la conduite efficace de pratiques, d'où leur importance dans les actions des praticiens. Comme Hofstetter et Schneuwly (2009), ces auteurs perçoivent les CIR comme des savoirs en constante évolution qui exigent des habiletés spécifiques pour les acquérir, voire une compréhension de leur rôle et de leur utilité.

Ces auteurs ne sont d'ailleurs pas les seuls à parler d'habiletés ou d'aptitudes nécessaires à l'utilisation des CIR. Ainsi, pour Hemsley-Brown (2004), les CIR s'associent à des informations précieuses qui permettent de réduire les risques de mauvaises interventions lors de prise de décisions en contexte éducatif. Plus précisément, ces auteurs conçoivent les CIR comme des données probantes à la fois nécessaires et utiles aux praticiens lors de processus décisionnels. Des données qui peuvent être utilisées directement par les acteurs scolaires en place, moyennant l'acquisition de quelques habiletés et compréhensions spécifiques.

En somme, il émerge dès lors une première conception des CIR en tant que connaissances mobilisables, exploitables dans l'action par les acteurs scolaires, acteurs qui auront été outillés en termes d'habiletés. Il s'agit d'une conception qu'on peut d'emblée opposer à une seconde: celle où les CIR ne seraient pas des connaissances créées pour les intervenants scolaires, dans le sens où elles peuvent difficilement être exploitables ou utilisables dans l'action.

De ce fait, pour Hemsley-Brown et Sharp (2003) ainsi que Kirst (2000), la plupart des CIR, telles qu'elles sont généralement produites - selon le contexte des chercheurs, des scientifiques - ne peuvent être utilisées directement par les acteurs scolaires. Selon ces auteurs, bien que les connaissances issues de la recherche devraient être applicables au contexte éducatif des utilisateurs, ce n'est pas le cas actuellement. Si les auteurs ne précisent pas comment ils conçoivent les connaissances actuellement produites en éducation, ils explicitent "ce que devraient être» idéalement les CIR en éducation au regard de leur usage. En ce sens, les CIR devraient être des connaissances plus contextualisées, valides, de façon à être utilisables immédiatement par les utilisateurs.

Aboulaye (2003) ainsi que Abrami et al. (2010) abondent dans ce sens alors qu'ils conçoivent les CIR comme des connaissances visant à distinguer les bonnes pratiques à 
exploiter selon des contextes situationnels scolaires précis. Il s'agirait d'une connaissance semblable à celle que l'on retrouve en médecine: au vu de tel symptôme (difficulté, besoin observé) s'orientant sur tel diagnostic (problème entrevu), les actions possibles sont les suivantes pour agir de manière efficace. Ce dernier postulat rejoint en partie celui de Lemire et al., (2011) pour qui les CIR relèvent globalement de constats empiriques de recherche. Or, selon ces auteurs, les connaissances créées conjointement entre chercheurs et praticiens seraient véritablement au service des praticiens.

\subsection{Le transfert des connaissances}

Selon Gervais, Marion, Dagenais, Chiocchio et Houlfort (2015) qui ont réalisé une étude sur la terminologie du TC, il n'existe aucun consensus en termes de nomenclature entourant le concept de transfert des connaissances. Toutefois, ces auteurs rapportent qu'en regroupant les domaines de la santé ainsi que des sciences humaines et sociales, le terme le plus employé dans l'ensemble des écrits portant sur le thème du TC est celui de "transfert des connaissances". En effet, leurs travaux rendent compte de la préférence des auteurs de ces domaines pour le terme "transfert des connaissances" avec $43 \%$ d'occurrences, suivi par "mobilisation des connaissances" avec $27 \%$ d'occurrences, puis "utilisation des connaissances» avec 7 \% d'occurrences (Ibid.). C'est pourquoi nous avons retenu le terme "transfert des connaissances" dans le présent texte.

Selon Trevithick-Oglesby (2008), le transfert des connaissances est une pratique qui englobe la production de connaissances - sous une forme utilisable - suivie de sa diffusion jusqu'à son utilisation. Lui préférant le concept “mobilisation des connaissances” (knowledge mobilization), Cooper et Levin (2013) définissent le phénomène comme des efforts fournis intentionnellement pour accroitre l'utilisation de données de recherche (données recueillies par le biais de processus formels systématiques et d'enquêtes ayant fait l'objet de travaux empiriques plutôt que de simples études) pour l'adoption de politiques et de pratiques efficaces à différents niveaux du secteur de l'éducation. Leur conception rejoint celle du Fonds de recherche du Québec - Société et culture (FRQSC) (2011) qui définit le transfert des connaissances comme "[I']ensemble des efforts consentis pour contribuer à faire connaitre et reconnaitre les activités et les résultats de recherche en sciences sociales et humaines, en arts et en lettres en vue de leur utilisation par les milieux de pratique, les décideurs et le grand public, que la démarche soit interactive ou non» (FRQSC, 2011, p. 9) 
Cette question de démarche interactive dans la définition du TC, si elle peut être présente ou non pour le FRQSC (2011), s'avère essentielle pour d'autres auteurs. Par exemple, pour Lemire et al. (2011), le transfert des connaissances réfère à un échange de savoirs entre chercheurs et utilisateurs. Ces derniers précisent que cet échange doit être compris comme une communication de la connaissance des agents producteurs - les chercheurs - aux agents utilisateurs que sont les praticiens. Idéalement, cet échange s'inscrit dans un processus composé de véritables interactions entre chercheurs et utilisateurs, le tout impliquant coopération et apprentissage mutuel. Cela, considérant que d'autres moyens peuvent être avancés pour la communication des connaissances tels l'utilisation de documents, le web, les médias, etc. (Lemire et al., 2011).

Pour d'autres auteurs comme Hemsley-Brown (2004), la dimension d'interactivité doit prendre en compte la réalité et les besoins des utilisateurs. Pour ce dernier, le transfert des connaissances ne peut être défini sans mention de ces aspects implicites à la considération de l'utilisateur, soit: 1) la source de dissémination (d'où proviennent les connaissances); 2) le produit disséminé (pertinence pour utilisateurs, qualité, sens, etc.); 3) les utilisateurs (leur motivation à vouloir les utiliser); 4) les canaux de communication; 5) le format de communication choisi (langage, forme, etc.); et 6) les ressources et moyens affectés pour ces activités. Ces postulats impliquent des étapes qui vont au-delà de la simple dispersion de connaissances d'un milieu à un autre par la mise en œuvre de moyen de diffusion.

En somme, les conceptions du TC varient en termes d'étapes et de dimensions impliquées. Ce qui n'est pas étonnant puisque selon Dagenais et al., (2008) ainsi que Gervais et al. (2015), il existe une confusion entourant le concept de transfert de la connaissance. Plusieurs définitions traitent ainsi de dimensions sous-jacentes au transfert, ces dernières semblables ou divergentes selon les postulats: place accordée aux activités de production et de diffusion des connaissances, type de connaissances à prioriser, degré d'importance allouée à l'interactivité ou au partage de savoirs dans le processus (Gervais et al., 2015). D'ailleurs, selon les domaines de recherche et les auteurs, le concept est défini en des termes différents: "transfert", "utilisation", "mobilisation", "application", "valorisation", "échange", etc. "des connaissances". "Si des perspectives s'observent plus particulièrement en lien avec certains termes et leur conceptualisation, la littérature sur le TC rend compte» de concepts et de définitions utilisés de façon interchangeable en fonction des visions de chacun (Ibid.).

En définitive, s'il faut concevoir le concept de transfert de connaissance selon les dimensions implicites au processus, il n'est pas étonnant qu'il y ait un manque de 
ressources et de moyens, voire de temps investi, tant du côté des chercheurs en milieu universitaire qui produisent la connaissance que du côté des utilisateurs à même d'en bénéficier. C'est du moins l'une des conclusions du rapport de Brodeur, Fontan, Landry, Auclair et Tirilly (2009). Nous conclurons à ce stade que le concept de transfert des connaissances semble effectivement dépasser largement la simple dissémination de données d'un milieu à un autre. En ce sens, le TC paraît englober toutes les étapes allant de sa production (incluant les visées et intentions des producteurs) à la phase d'intégration desdites connaissances par les utilisateurs (sous-jacente à des changements tangibles dans leurs actions). La prise en compte des utilisateurs dans le processus de TC ainsi que l'espace accordé à l'interactivité paraissent également des dimensions incontournables.

\section{Méthodologie}

Nos résultats découlent d'une recension des écrits sur le TC en éducation et dans les principaux domaines nommés ci-avant. Considérant la revue systématique des écrits sur le TC en éducation produite par Landry et al. en 2008, seuls les textes parus entre 2007 et 2013 ont été retenus afin de réaliser un état des lieux plus actuel. Précisons ici que les principales conclusions de cette revue systématique rendent compte de l'importance de référer à des agents de liaison pour établir des ponts entre les milieux de recherche et de pratique et ainsi faciliter le transfert des CIR. La revue aborde aussi des déterminants à considérer pour accroitre le TC concernant: 1) l'utilisateur ou le gestionnaire (scolarité, expérience en TC, facteurs personnels, capacité cognitive, motivation, temps disponible, capital relationnel); et 2) l'organisation (caractéristiques, ressources, contacts, politiques favorisant le TC, climat, gestion du changement, clarté des demandes et besoins, types de formation privilégiés). Les auteurs évoquent finalement la nécessité que les utilisateurs soient impliqués à toutes les étapes du processus de TC, que les CIR soient adaptées en fonction des besoins et attentes du public cible, et que les interactions entre chercheurs, accompagnateurs et utilisateurs soient continues (Landry et al., 2008).

En retraçant différents écrits suivant l'année 2007, la visée est alors de brosser un portrait des avancées produites en éducation, comparativement à l'état d'avancement de la recherche dans l'ensemble des domaines actifs sur le thème du TC (santé, sciences sociales, appliquées, psychosociales, gestion, milieux universitaires).

Nous avons choisi comme méthodologie le scoping study (Arksey et O’Malley, 2005; Levac, Colquhoun et O'Brien, 2010). Plus connue dans le domaine de la santé, le scoping study est une méthode de recherche de plus en plus utilisée ces dernières 
années pour des études visant à dresser une cartographie, soit une synthèse d'un objet de recherche circonscrit (Levac et al., 2010). La méthode consiste en une étude de cadrage orientée autour de concepts clés en vue d'obtenir le portrait global des publications autour d'une question ou d'un domaine de recherche. Selon Arksey et O'Malley (2005), le scoping study se révèle utile pour: 1) déterminer la mesure, le caractère ou la nature d'un objet de recherche; 2) identifier la pertinence de réaliser une revue systémique; 3) diffuser des résultats de recherche ciblés et 4) repérer les lacunes d'une littérature de recherche. Nous souscrivons pour notre part à la première et à la quatrième visée. Nous comptons en ce sens situer la nature et les caractéristiques du TC en éducation en dressant un portrait global de la situation, pour mieux relever les manques à combler.

La recension a été réalisée à partir des bases de données de recherche suivantes: ERIC, MEDLINE, PsycINFO, BDSP, FCRSS, EBSCO, ProQuest Dissertations and Theses, CAIRN, Érudit et Google Scholar. Les mots clés utilisés en référence au TC pour le repérage des articles furent les suivants:

Transfert + connaissance / savoir / donnée de recherche
Mobilisation + connaissance / savoir / donnée de recherche
Utilisation + connaissance / savoir / donnée de recherche
Application + connaissance / savoir / donnée de recherche
Dissémination + connaissance / savoir / donnée de recherche
Diffusion + connaissance / savoir / donnée de recherche
Gestion + connaissance / savoir / donnée de recherche
Knowledge / research results / research / evidence + transfer
Knowledge / research results / research / evidence + utilization
Knowledge / research results / research / evidence + exchange
Knowledge / research results / research / evidence + management
Evidence based / evidence-based / evidence use + practice

Un total de 181 publications (de provenance canadienne et d'autres pays) ont été relevées dans un premier temps. De ce nombre, 51 sources ont été retirées en raison de critères d'exclusion (voir tableau 1). Au final, 132 publications ont été retenues pour le traitement et l'analyse des données.

\subsection{Critères d'inclusion et d'exclusion}

Conformément à la méthodologie proposée par Arksey et O'Malley (2005), la deuxième étape de notre étude consistait à identifier et à appliquer des critères d'inclusion et d'exclusion. Ils sont présentés dans le tableau qui suit: 
Tableau 1

Critères d'inclusion et d'exclusion appliqués aux documents recensés sur le TC

\begin{tabular}{|l|l|}
\hline Critères d'inclusion & Critères d'exclusion \\
\hline Publié entre 2007 et 2013 & Publié avant 2007 \\
\hline $\begin{array}{l}\text { Sciences sociales, psychosociales, } \\
\text { appliquées, éducation, santé, gestion } \\
\text { ou sur au moins un de ces domaines } \\
\text { concernant le lien université et milieu } \\
\text { de pratique }\end{array}$ & $\begin{array}{l}\text { Domaine non ciblé par l'étude ou qui } \\
\text { ne concerne pas les utilisateurs ou le } \\
\text { milieu pratique }\end{array}$ \\
\hline Langue française et anglaise & Langue autre \\
\hline $\begin{array}{l}\text { Document accessible dans les } \\
\text { bibliothèques du réseau UQ }{ }^{2}\end{array}$ & $\begin{array}{l}\text { Coûts trop élevés, absence de lieux } \\
\text { physiques ou virtuels de retrait }\end{array}$ \\
\hline $\begin{array}{l}\text { Article scientifique, chapitre ou } \\
\text { ouvrage découlant d'une } \\
\text { méthodologie scientifique, rapport de } \\
\text { recherche, essai, maîtrise et thèse }\end{array}$ & $\begin{array}{l}\text { Article professionnel, compte rendu } \\
\text { de colloque, commentaire et texte } \\
\text { non associé à une méthodologie } \\
\text { scientifique }\end{array}$ \\
\hline $\begin{array}{l}\text { Résultats de recherche empirique, } \\
\text { systématique ou de revue critique ou } \\
\text { exploratoire }\end{array}$ & $\begin{array}{l}\text { Méthodologie non précisée, résultats } \\
\text { ne relevant pas de critères } \\
\text { scientifiques }\end{array}$ \\
\hline
\end{tabular}

${ }^{2}$ Réseau de I'Université du Québec qui compte 10 établissements.

L'application de ces critères à l'étape 1 nous a permis de centrer notre recherche sur 132 textes. Le tableau suivant rend compte des types de publication et de recherche que comportait le corpus de textes. 
Tableau 2

Répartition des 132 sources selon le domaine, le type de publication et de recherche

Nombre de sources selon le domaine

\begin{tabular}{|c|c|c|}
\hline 41 Éducation & $\begin{array}{l}24 \mathrm{AS} \\
5 \mathrm{C} \\
8 \mathrm{RR} \\
4 \mathrm{EMT}\end{array}$ & $\begin{array}{l}1 \mathrm{RS} \\
21 \mathrm{ECC} \\
19 \mathrm{RE}\end{array}$ \\
\hline $\begin{array}{l}32 \text { Social et } \\
\text { psychosocial }\end{array}$ & $\begin{array}{l}18 \text { AS } \\
5 \mathrm{C} \\
7 \mathrm{RR} \\
2 \mathrm{EMT}\end{array}$ & $\begin{array}{l}4 \mathrm{RS} \\
15 \mathrm{ECC} \\
13 \mathrm{RE}\end{array}$ \\
\hline 27 Santé & $\begin{array}{l}20 \text { AS } \\
3 \mathrm{C} \\
2 \mathrm{RR} \\
2 \mathrm{EMT}\end{array}$ & $\begin{array}{l}4 \mathrm{RS} \\
9 \mathrm{ECC} \\
14 \mathrm{RE}\end{array}$ \\
\hline 19 Gestion & $\begin{array}{l}12 \text { AS } \\
2 \mathrm{C} \\
3 \mathrm{RR} \\
2 \mathrm{EMT}\end{array}$ & $\begin{array}{l}1 \mathrm{RS} \\
12 \mathrm{ECC} \\
6 \mathrm{RE}\end{array}$ \\
\hline $\begin{array}{l}8 \text { Lien universités et } \\
\text { milieux de pratiques }\end{array}$ & $\begin{array}{l}6 \text { AS } \\
2 \text { RR }\end{array}$ & $\begin{array}{l}5 \mathrm{ECC} \\
3 \mathrm{RE}\end{array}$ \\
\hline 4 Sciences appliquées & 4 AS & $\begin{array}{l}2 \mathrm{ECC} \\
2 \mathrm{RE}\end{array}$ \\
\hline
\end{tabular}

Type de publication Type de recherche

\section{Type de recherche}

Légende: $\mathrm{AS}=$ article scientifique; $\mathrm{C}=$ chapitre ou ouvrage impliquant méthodologie; $\mathrm{RR}=$ rapport de recherche; EMT = essais, mémoire ou thèse; $\mathrm{RS}=$ revue systématique; $E C C=$ recherche exploratoire, revue critique ou scoping study; $\mathrm{RE}=$ recherche empirique. 


\subsection{Analyse}

L'analyse de textes dans le cadre d'un scoping study vise à synthétiser et à organiser le matériel recensé selon des thèmes spécifiques (Arksey et O'Malley, 2005). Ce processus est itératif, c'est-à-dire que, bien que des thèmes prédéfinis ont servi à codifier les documents lus, d'autres ont émergé et ont été ajoutés pour raffiner les analyses. L'analyse consistait à identifier des extraits, à les classifier dans des thèmes, à relever le dénombrement des thèmes abordés dans les textes (citations), pour ensuite procéder à l'analyse de ces citations. Les thèmes retenus (thèmes prédéfinis: thèmes définis avant de procéder à l'analyse; thèmes émergents: thèmes apparus en cours d'analyse) et le nombre de citations sont répertoriés dans le tableau 3.

Tableau 3

Thèmes retenus et nombre de citations obtenu

\begin{tabular}{|c|c|}
\hline Thèmes prédéfinis & Thèmes émergents \\
\hline Définition du TC (67) & $\begin{array}{l}\text { - Cadre théorique (concepts reliés) (11) } \\
\text { - Historique (31) }\end{array}$ \\
\hline \multicolumn{2}{|l|}{ Utilité ou importance du TC (91) } \\
\hline \multicolumn{2}{|l|}{ Défis et enjeux du TC (72) } \\
\hline Types de connaissances (108) & $\begin{array}{l}\text { - Utilité, rôle (38) } \\
\text { - Défi relié (61) }\end{array}$ \\
\hline \multicolumn{2}{|l|}{ Type d'utilisations des CIR (56) } \\
\hline Entraves au TC & $\begin{array}{l}\text { - Endogènes (84) } \\
\text { - Physiques (8) } \\
\text { - Culturels (19) } \\
\text { - Organisationnels (74) } \\
\text { - Milieux de recherche (53) }\end{array}$ \\
\hline Facilitateurs au TC & $\begin{array}{l}- \text { - Endogènes (45) } \\
\text { - Physiques (8) } \\
\text { - Culturels (13) } \\
\text { - Organisationnels (102) } \\
\text { - Milieux de recherche (48) }\end{array}$ \\
\hline Stratégies, approches, modèles (317) & $\begin{array}{l}\text { Courtage de connaissances } \\
\text { - définition, stratégies, tâches, types, } \\
\text { impacts - (224) }\end{array}$ \\
\hline \multicolumn{2}{|l|}{ Étapes ou processus (71) } \\
\hline Impacts du TC (83) & \\
\hline
\end{tabular}


Le logiciel NVivo a été utilisé pour faciliter les analyses. Une fois les citations relevées, un travail de structuration des données puis de synthèse a été réalisé pour clarifier et exploiter le contenu de chacun des thèmes. La dernière étape de ce travail a consisté à relever les convergences obtenues pour chacun des thèmes en lien avec tous les domaines regroupés. L'exercice a été répété pour les citations dérivant des écrits issus de l'éducation de manière à obtenir les convergences pour ce domaine distinct.

\section{Résultats}

Les passages suivants rendent compte des principaux résultats de notre étude. Ils sont abordés selon un regard croisé: présentation de la situation globale et de celle qui prévaut plus particulièrement en éducation.

\subsection{Le TC: un concept à visage multiple}

Qu'est-ce que le TC? Les résultats du scoping study réalisé révèlent que le concept est évoqué en des termes différents - "transfert", "mobilisation", "utilisation", "application", "dissémination", "partage des connaissances", etc. - qui incluent notamment des divergences dans l'implication, la responsabilité et le rôle des chercheurs qui produisent les CIR (Dagenais et al., 2008). Le concept est également souvent défini selon les étapes allant de la production de connaissances à leur utilisation par les utilisateurs (professionnels ou décideurs). Nos résultats d'analyse indiquent que la définition du TC s'inscrit dans deux perspectives ou visions principales. La première réfère à un processus bidirectionnel où producteurs et utilisateurs s'échangent des connaissances en vue d'en venir à l'élaboration de connaissances contextualisées. Ici, la définition du TC traduit la place importante accordée à l'interactivité, mais également aux utilisateurs, à leurs connaissances tacites (savoirs d'expérience) et à leur contexte de travail. La deuxième perspective réfère à un processus itératif ou non entre producteurs et utilisateurs orienté sur le déploiement de stratégies afin que des CIR puissent être transférées dans des organisations jusqu'à leur utilisation. Si cette perspective peut impliquer une prise en compte des utilisateurs (valeurs, connaissances d'expérience, milieu), il peut aussi s'agir simplement d'user de moyens opérationnels pour que les utilisateurs transfèrent des CIR et les intègrent à leurs pratiques.

En éducation, malgré l'utilisation de termes divers, les définitions s'inscrivent davantage à l'intérieur des trois visions suivantes: 1) certains auteurs référent tout simplement à l'idée de connexion ou de mise en liens entre la recherche et la pratique 
(par ex., Broekkamp et Van Hout-Wolters, 2007; Edelstein, 2011; Flessner, 2012); 2) d'autres définissent le concept comme un processus interactif qui implique différents moyens et efforts pour accroitre l'utilisation des connaissances par les praticiens (par ex., Abrami et al., 2010; Cooper, 2013; Sa et al., 2010); 3) d'autres encore, comme Biasutti et El-Deghaidy (2012) et Spencer, Detrich et Slocum (2012) le décrivent plus particulièrement comme un processus de partage et de création grâce auquel des apprenants organisent et intègrent des connaissances explicites - internalisation - dans des connaissances tacites.

\subsection{Types d'utilisation des CIR et leurs effets}

Notre analyse révèle cinq types d'utilisation des connaissances. II y aurait: 1) I'utilisation instrumentale qui consiste à recourir aux CIR pour la prise de décisions de changement de pratiques selon un mode linéaire et à court terme (Gera, 2012); 2) I'utilisation conceptuelle qui a trait à l'usage de CIR orientées sur une nouvelle façon de voir le monde, en lien avec une conscientisation accrue ou l'approfondissement d'une compréhension (Meagher, 2013) et 3) l'utilisation persuasive ou symbolique qui vise à recourir aux CIR d'un point de vue stratégique pour influencer des choix ou encore confirmer une pratique (Abrami et al., 2010). Plus récemment, Lemire, Souffez et Laurendeau (2009) ont abordé 4) l'utilisation processuelle qui "fait référence à l'impact du processus de la recherche sur les participants" (p. 25). Ainsi, seulement par leur implication dans un processus de recherche, des participants peuvent modifier leurs façons de penser ou leurs pratiques. Pour leur part, Elissalde, Gaudet et Renaud (2010) ont ajouté 5) l'utilisation inspirante (ou indirecte pour Osterling et Austen, 2008) comme sous-thème à l'utilisation conceptuelle. Il s'agit pour des utilisateurs de transposer des CIR à un domaine complètement différent.

En ce qui concerne les effets de ces types d'utilisation, les recherches ont surtout documenté les effets proximaux (effets sur les utilisateurs ou le milieu proche) découlant de l'utilisation instrumentale (par ex., Martin, Jensen, Coatsworth-Puspoky, Forchuk, Lysiak-Globe et Beal, 2007). Ces effets se traduisent par des modifications de comportement chez des praticiens et des décideurs. L'utilisation instrumentale aurait des effets positifs sur les habiletés, les attitudes et les façons de penser. Notre analyse indique que les effets distaux des différentes formes d'utilisation des CIR - soit les impacts sur les clientèles impliquées - sont rarement étudiés. Enfin, nous n'avons identifié aucune étude sur les effets proximaux et distaux des utilisations conceptuelle et persuasive. 
Lestroisformesprincipalesd'utilisation-«instrumentale”, "conceptuelle» et«persuasive»seraient présentes dans les milieux scolaires (Becheikh et al., 2010). Elles agiraient souvent en interrelation et simultanément (Abrami et al., 2010). De plus, si les décideurs ont surtout recours à l'utilisation instrumentale (pour des critères de performance et l'élaboration d'objectifs), les enseignants privilégient l'utilisation conceptuelle (Dagenais, Janosz, Abrami et Bernard, 2007). Les enseignants recourent plus particulièrement aux CIR pour satisfaire leur curiosité, créer des activités ou du matériel scolaire (Dagenais et al., 2007). Par ailleurs, il semble que les conseillers pédagogiques utilisent essentiellement les CIR pour solutionner des problèmes de pratique, parfaire leur compréhension ou justifier des décisions: ce qui donne lieu à une utilisation instrumentale, conceptuelle et persuasive. Notons finalement que l'utilisation symbolique ou persuasive se verrait davantage chez les administrateurs scolaires pour légitimer le recours à certaines pratiques ou justifier des décisions politiques (Edelstein, 2011).

\subsection{Le processus de TC}

Différents auteurs ont cherché à rendre compte du processus de TC, tantôt évoqué selon des questions, tantôt selon des étapes ou des variables impliquées. Lemire et al. (2011) évoquent ainsi le processus de Lavis, Robertson, Woodside, McLeod et Abelson (2003) qui abordent le TC selon cinq questionnements: quoi? pour qui? par qui? comment? est-ce que ça fonctionne? Pour sa part, Souffez (2012) recense huit étapes au TC: 1) la production, 2) l'adaptation, 3) la diffusion, 4) la réception, 5) l'adoption, 6) I'appropriation, 7) l'utilisation des connaissances et 8) l'appréciation des retombées.

Le processus de TC s'avère dynamique, en plus d'être sous l'influence de multiples variables dont les caractéristiques individuelles des utilisateurs, celles de l'organisation et des politiques en vigueur (Winton et McCollum, 2008). Comme autres variables, il faut ajouter les caractéristiques reliées à l'objet de TC qu'est la connaissance (Chagnon et Gervais, 2011), aux producteurs de CIR (Becheikh et al., 2010; Cloutier, 2010) ainsi que celles associées au monde de la recherche (Sa et al., 2010; St-Cyr Tribble et al., 2008).

\subsection{Les modèles de TC}

Pour Abrami et al. (2010), l'ensemble des modèles de TC vise à expliquer la complexité du processus de transfert. II en existerait plus d'une soixantaine et aucun n'emporte l'adhésion générale des chercheurs (Chagnon, Daigle, Gervais, Houle et Béguet, 2009; Dagenais et Robert, 2012). Pour Dagenais (2012), les trois types de modèles les plus documentés dans la littérature sont: 1) le "science push" selon lequel 
une connaissance pertinente est utilisée sans intervention spécifique, 2) le "demand pull" qui réfère à l'utilisateur manifestant un besoin à des chercheurs en vue de résoudre un problème et 3) le modèle de "l'interaction sociale» qui implique une collaboration entre chercheurs, décideurs et utilisateurs à toutes les étapes du transfert. En santé, il appert que le science push model (ou académique) serait encore prédominant (Abrami et al., 2010). Or, comme plusieurs études ont démontré les limites du science push model, de plus en plus d'auteurs invitent à utiliser le modèle d'interaction sociale pour faciliter le TC (par ex., Abrami et al., 2010; Chagnon et al., 2009; Elissalde et al., 2010). Par ailleurs, des données plus fines révèlent qu'étant donné la diversité des contextes de travail d'un domaine à l'autre, un modèle de TC élaboré pour l'un ne conviendrait pas nécessairement à un autre (Chagnon et Gervais, 2011; Souffez, 2012).

En éducation, le modèle présumé le plus efficace par certains auteurs est celui de l'interaction sociale (par ex., Beckeih et al., 2010; Levin et al., 2011; Nelson, Leffler et Hansen, 2009).

\subsection{Déterminants à prendre en compte dans le TC}

Notre analyse a permis de détailler tant des leviers que des entraves, stratégies et déterminants du TC. Or, comme plusieurs leviers et entraves concernent souvent un même aspect (selon un pôle positif ou négatif) et que des stratégies sont parfois évoquées comme des déterminants et vice-versa, il a été décidé de référer à chacun d'eux par un même concept: les déterminants du TC. Le texte qui suit présente ceux qui sont les plus évoqués dans les publications.

\subsubsection{Système et contexte organisationnel}

Alors qu'au début du deuxième millénaire, la tendance était d'entreprendre des actions auprès des utilisateurs de manière à ce qu'ils changent graduellement la culture organisationnelle, aujourd'hui, c'est plutôt l'inverse. De plus en plus d'auteurs considèrent que c'est en modifiant les aspects organisationnels d'un milieu que ses utilisateurs s'inscrivent dans un meilleur TC (par ex., Davies et Nutley, 2008; Florin, Ehrenberg, Wallin et Gustavsson, 2011; Harvey, 2010; Levin et al., 2011). Au terme d'une étude réalisée auprès d'infirmières, Florin et ses collaborateurs (2011) ont révélé que, sans modification dans la culture organisationnelle, une hausse des compétences individuelles à l'évaluation critique de CIR n'entraine pas un meilleur TC. Plus précisément, il serait judicieux d'avoir recours à une combinaison de stratégies et de moyens visant à développer une culture du TC: création de réseaux, ajout d'intermédiaires, instauration de mesures de soutien 
et de communication, activités professionnelles axées sur le TC, etc. (Landry et al., 2008; Nelson et al., 2009; St-Cyr Tribble et al., 2008). Il peut s'agir également de chercher à multiplier les lieux d'échange et de rencontre entre producteurs de CIR et utilisateurs (Bammer, Michaux et Sanson, 2010; Gervais et Chagnon, 2010; Lacroix, 2012; Ziam, 2010). L'élaboration d'un corpus des meilleures pratiques de l'organisation est aussi conseillée (Bourdeau, 2011; Gera, 2012; Lemire et al., 2011).

Par ailleurs, plusieurs auteurs insistent sur la nécessité que du temps et des plages horaires soient dégagés pour les activités de TC (Belleau, 2011; Lemire et al., 2011; Harvey, 2011; Nelson et al., 2009). D'autres abordent le recours à des plateformes ou à des applications Internet destinées à soutenir le transfert de CIR, dont le Web 2.0 (Edelstein, 2011; Paroutis et Saleh, 2009). Enfin, des résultats soulignent l'importance que les gestionnaires et décideurs soient non seulement sensibilisés au TC, mais impliqués dans les activités prévues à cet effet (Bourdeau, 2011; Collerette, 2008; Crona et Parker, 2011; Landry et al., 2008). Leur compréhension et leur apport auraient un impact tant sur la mobilisation des utilisateurs que sur l'instauration d'une culture de recherche dans le milieu.

Finalement, le recours à des courtiers de connaissances - ou agents de liaison est particulièrement évoqué ces dernières années comme stratégie innovatrice du TC pour les organisations (Crona et Parker, 2011; Lamari, 2012; Long, Cunningham et Braithwaite, 2013; Meyer, 2010; Nelson et al., 2009; Osterling et Austin, 2008; Ridde, Dagenais et Boileau, 2013; Ward, House et Hamer, 2009; Williams et al., 2008; Ziam, 2010). Dans le domaine de la santé ainsi que des sciences sociales et psychosociales, l'utilisation de courtiers de CIR est d'ailleurs commentée dans plusieurs études. Bien que son rôle puisse varier, le courtier de connaissances sert d'abord d'interface entre producteurs de CIR et utilisateurs (Lamari, 2010). Au vu des besoins, les tâches qui lui sont attribuées peuvent être restreintes et circonscrites ou multiples: gestion de l'information via des sites de veille, recension, sélection et diffusion des CIR, vulgarisation, production de synthèses, orchestration de rencontres et d'activités de TC, réseautage, accompagnement, soutien aux utilisateurs, liaison entre chercheurs, utilisateurs, décideurs et autres acteurs, etc. (Bammer et al., 2010; Ridde et al., 2013).

En éducation, Chabot (2007) indique que les principales entraves au TC sont de nature organisationnelle. Aussi, pour améliorer la situation, plusieurs chercheurs recommandent des modifications importantes sur ce plan. Il est question de multiplier les interfaces entre producteurs et utilisateurs avec l'ajout de courtiers de connaissances 
et d'organismes jouant le rôle d'intermédiaire (Beckeih et al., 2010; Dagenais et al., 2007; Landry et al., 2008; Levin et al., 2011; Nelson et al., 2009; Shonkoff et Bales, 2011; Tseng, 2012). Ce courtier peut être un professionnel de recherche ayant la formation nécessaire ou encore un chercheur adoptant ce rôle. Aussi, dès l'embauche, les acteurs scolaires pourraient bénéficier de formations portant sur l'évaluation, la pertinence et I'utilisation de la recherche (Abrami et al., 2010; Chabot, 2007; Dagenais et al., 2007; Ramdé, 2012). L'implantation de stratégies de circulation et de communication des CIR (bulletins d'information, sites Internet, rencontres informelles, suivis de formation de TC, activités et événements encourageant le TC) pourrait également s'avérer profitable (Abrami et al., 2010; Chabot, 2007; Levin, 2010). En outre, il importe de chercher à mettre en place des conditions favorisant un meilleur TC pour les utilisateurs (Chabot, 2007; Bricout, Pollio, Edmond et McBride 2008; Dagenais et al., 2007; Nelson etal., 2009; Ramdé, 2012; Shonkoff et Bales, 2011). Selon ces auteurs, il peut s'agir de: 1) temps supplémentaire octroyé, 2) mesures de reconnaissance pour activités de TC ou ajout à la description de tâche, 3) sensibilisation et implication des décideurs scolaires, 4) gestion participative, 5) mesures de soutien et d'accompagnement s'inscrivant sur le long terme, 6) décisions et orientations politiques orientées sur le TC, 7) ajout ou repositionnement de personnes clés.

\subsubsection{Prise en compte et accompagnement des utilisateurs}

Plusieurs résultats d'études incitent à reconsidérer la place et l'importance des utilisateurs dans le processus. Certaines caractéristiques des utilisateurs seraient prépondérantes: leurs valeurs, points de vue, besoins et ce qui est signifiant pour eux (Bammer et al., 2010; Belleau, 2011; Cloutier, 2010; Dagenais et al., 2008; Hotho, Becker-Ritterspach et Saka-Helmhout, 2012; Osterling et Austin, 2008). Pour transférer les CIR, les utilisateurs auraient besoin d'écoute, d'échanges continus et structurés qui s'inscrivent dans le long terme (Chagnon et al., 2009; Lemire et al., 2011; St-Cyr Tribble et al., 2008). À cet effet, on devrait privilégier des rencontres en face à face (Ridde et al., 2013) prenant parfois la forme de coaching tout en recourant à des personnes crédibles. Celles-ci seront respectées en tant que spécialistes et agiront comme leadeurs dans le processus (Ash et al., 2012; Lemire et al., 2011). En outre, on optera pour des rapports égalitaires fondés sur l'optique que chacun apporte sa contribution au processus (Belleau, 2011; St-Cyr Tribble et al., 2008). Le développement de relations de qualité, de confiance entre partenaires est aussi à considérer (Elissalde et al., 2010; Lemire et al., 2011). Finalement, pour des auteurs, il y a une nécessité que les utilisateurs soient impliqués dans toutes les étapes du processus de TC (Belleau, 2011; Cloutier, 2010; Gervais et Chagnon, 2010; Lemire et al., 2011). Une telle implication favoriserait l'appropriation des 
connaissances par les utilisateurs, car le processus aura allié coconstruction, collaboration, prise en compte des besoins et recherche de sens.

En éducation plus particulièrement, des auteurs évoquent l'importance d'une équipe stable et d'une direction à l'écoute qui exercent un leadeurship favorisant le TC (Chabot, 2007; Dagenais et al., 2007; Nelson et al., 2009). Pour Presse (2009), le TC doit impliquer la volonté des différents intervenants scolaires ainsi qu'un partage de valeurs avec les chercheurs. Ainsi, outre les enseignants, il importe que les membres formant l'équipe de direction et les décideurs soient non seulement sensibilisés, mais engagés dès les premières rencontres (Dagenais et al., 2008). Comme l'indique Chabot (2007), "bien qu'il ait été démontré que la recherche permet de favoriser le succès scolaire des élèves, la responsabilité de I'utilisation des CIR n'incombe pas uniquement aux enseignants" (p. 40). Les différents acteurs auraient aussi besoin d'interactions fréquentes qui s'inscrivent dans le temps (Presse, 2009; Ramdé, 2012; Sylva, Taggart, Melhuish, Sammons et Siraj-Blatchford, 2007; Tseng, 2012; Veillard et Coppé, 2009). Certains parlent même de collaboration permanente entre chercheurs et acteurs scolaires (Biesta, 2007; Hedges, 2010). Enfin, les intervenants scolaires, et particulièrement les enseignants, auraient besoin d'être soutenus et que soient pris en compte leurs besoins et réalités (par ex., Chabot, 2007; Hedges, 2010; Nelson et al., 2009; Presse, 2009). Pour sa part, Cordingley (2008) croit en un processus d'apprentissage professionnel personnalisé aux besoins et au contexte des praticiens.

\subsubsection{Adaptation des connaissances}

Pour maints auteurs, il est nécessaire d'adapter les CIR de manière à favoriser leur mobilisation par les utilisateurs (par ex., Belleau, 2011; Gervais et al., 2011; Elissalde et al., 2010; Nelson et al., 2009). Il est question d'adaptation au vu du langage (vulgarisé), du format et suivant les besoins des utilisateurs (pertinence, contextualisation, valeurs). Au terme d'une thèse réalisée sur l'adaptation des résultats de la recherche en santé, Jbilou (2010) évoque sept formes d'adaptation: 1) sémantique (langage non technique), 2) didactique (avec exemples et démonstrations), 3) tactique (rapports illustrés, graphiques, schémas, formats colorés ou humoristiques), 4) dialogique (avec discussions et interactions prévues), 5) thématique (rapports sur des sujets précis), 6) électronique (bulletins d'information pour utilisateurs) et 7) stratégique (recours à des experts ou à des consultants). Jbilou invite à combiner certaines formes d'adaptation selon les besoins des utilisateurs. 
Elissalde et al. (2010) ainsi que Belleau (2011) croient, pour leur part, à une combinaison de moyens de diffusion: site Internet, page Facebook, blogues, service de veille documentaire, articles, rapports, etc. En outre, tel que mentionné dans les précédents passages, des auteurs abordent des processus (mise en récit, internalisation) qui favoriseraient l'intégration des connaissances explicites et tacites en vue de produire une nouvelle connaissance adaptée au contexte des utilisateurs (Abrami et al., 2010; Chagnon et al., 2009; Elissalde et Renaud, 2010). Il y aurait également un besoin d'adapter l'accessibilité ainsi que les délais de production et de diffusion des CIR (Chagnon etal., 2009).

Dans les milieux de l'éducation, des résultats de recherche témoignent de l'importance que les connaissances transférées soient rapides à intégrer, simples à comprendre, utiles, pertinentes, applicables directement et qu'elles s'alignent sur des besoins actuels (par ex., Abrami et al., 2010; Dagenais et al., 2012; Landry et al., 2008; Gera, 2012; Lin et Chen, 2009; Nelson et al., 2009; Ramdé, 2012; Saussez et Lessard, 2009). Les médias écrits, publications officielles, livres et ouvrages de référence faciliteraient le TC en éducation (Dagenais et al., 2007; Levin, 2010). Les enseignants en particulier utiliseraient essentiellement les CIR permettant de résoudre des problèmes vécus en classe (Chabot, 2007). Ces derniers privilégieraient les résultats présentés sous forme de lignes de conduite à adopter ou avec procédures détaillées (Abrami et al., 2010). De plus, les résultats de recherche devraient mieux expliciter le choix, la pertinence et les justifications d'une pratique évoquée; le "pourquoi» étant tout aussi important que le "quoi faire" (Crahay et al., 2010; Hedges, 2010). Les travaux de Dagenais et al. (2007) révèlent que les enseignants ressources et les conseillers pédagogiques utilisent plus souvent les TIC ${ }^{2}$ pour accéder aux CIR (comparativement aux enseignants et aux administrateurs). Pour leur part, les administrateurs scolaires préfèrent des données de recherche locale bien qu'ils en fassent un usage irrégulier. Concernant les TIC, Biasutti et El-Deghaidy (2012) croient que l'éducation doit se doter de Wiki afin que les acteurs scolaires puissent organiser et produire une connaissance qui réponde à leurs besoins, qui se traduirait par des pratiques et qui intègrerait des savoirs explicites et des savoirs tacites.

\subsubsection{Caractéristiques des utilisateurs pouvant faciliter le TC}

Certaines caractéristiques individuelles facilitent le TC chez les utilisateurs. II y a tout d'abord l'expertise en recherche des acteurs en lien avec leurs capacités, habiletés ou compétences à utiliser des CIR (par ex., Ouimet, Bédard et Lapointe, 2012; McCloskey, 2008; Mehiriz et Marceau, 2012). Dans cette optique, les aptitudes des 
utilisateurs à juger de leurs besoins, voire à évaluer la pertinence des CIR, joueraient un rôle important dans le TC (Gervais et Chagnon, 2012). Comme autres caractéristiques, on retrouve l'ouverture au TC, le degré d'initiative, l'esprit critique, la motivation des personnes (Osterling et Austen, 2008), de même que la confiance en soi (Gervais et Chagnon, 2010).

En éducation, des recherches évoquent l'ensemble des caractéristiques mentionnées ci-avant (par ex., Chabot, 2007; Dagenais et al., 2007; Mehiriz et Marceau, 2012; Ramdé, 2012). En ce qui a trait à l'expertise individuelle, Dagenais et al. (2007) ajoutent que "la majorité d'entre eux [parmi les intervenants scolaires] ne feraient pas la différence entre connaissance, information, stratégie, technique, pratique, donnée et statistique» (p. 10). Ils confondraient également les sources. De plus, un meilleur TC en milieu scolaire irait de pair avec des opinions, une attitude et des croyances favorables à la recherche (Dagenais et al., 2007). Comme autres caractéristiques pouvant augmenter le TC, Chabot (2007) relève le niveau de scolarité (plus élevé équivalant à un meilleur TC), le sentiment d'appartenance à l'école, le nombre de formations suivies et les préoccupations envers la réussite scolaire des élèves. Finalement, la capacité à lire l'anglais se révèlerait utile (les articles et les sites Internet anglophones étant plus nombreux) (Abrami et al., 2010).

\subsubsection{Caractéristiques des producteurs de CIR et des milieux de recherche}

Parmi les caractéristiques des producteurs de CIR qui favorisent le TC, on note la compréhension et la prise en compte des besoins et des réalités des utilisateurs (Bammer et al., 2010; Besner, Laurendeau et Bergeron, 2012; Lemire et al., 2011), ce qui se traduirait notamment par une ouverture à adapter les méthodes et les processus de recherche. Des capacités à collaborer et à dialoguer seraient tout aussi essentielles (Abrami et al., 2010; Brodeur et al., 2009; Dagenais et al., 2012). Des études évoquent également la crédibilité de la personne pour les utilisateurs ainsi que son habileté à adapter les CIR ou à recourir à des gens pour le faire (Beckeih et al., 2010; Dagenais et al., 2008; Ramdé, 2012). Il y aurait finalement la conscientisation à l'importance du TC dans I'amélioration des services à la population (Brodeur et al., 2009; Chagnon et Gervais, 2011; Dagenais et al., 2008).

En ce qui a trait aux milieux de recherche, des études abordent la nécessité de modifier certaines modalités et règles universitaires en vue de favoriser le TC (par ex., Brodeur et al., 2009; Dagenais et al., 2008; Geuna et Muscio, 2008; Gera, 2012). Il pourrait s'agir d'octroyer des incitatifs financiers aux producteurs pour accroitre les activités 
de TC (Abrami et al., 2010; St-Cyr Tribble et al., 2008; Sa et al., 2010), voire d'augmenter leur reconnaissance et valorisation dans la culture universitaire (Brodeur et al., 2009; Dagenais et al., 2008; Lacroix, 2012).

Pour ce qui est du domaine de l'éducation, nos analyses ne révèlent aucune donnée portant sur les producteurs de CIR et les milieux de recherche.

\section{Discussion}

L'objectif principal de cette étude est de situer le transfert des connaissances en éducation au regard de ce qui se passe globalement dans les autres domaines, cela en vue d'en clarifier les défis et les perspectives.

Les résultats de cette étude permettent de mettre en lumière certains constats. Pour commencer, il est intéressant de noter que des 132 documents analysés, seulement 57 rapportent des résultats d'études empiriques sur le TC (dont 19 en éducation). Les autres documents sont des revues de littérature systématiques, des revues critiques ou des études exploratoires. II semble donc que le champ de la recherche sur le TC, autant dans le domaine de l'éducation que dans les autres, soit en période de consolidation, ce qui reflète un besoin de circonscrire ses bases, de se (re)définir et d'identifier de nouvelles avenues de recherche prometteuses. Ce recul s'avère essentiel, surtout pour un champ en émergence où il y a une absence de taxonomie commune (concepts clés, déterminants et stratégies) et absence de modèle théorique intégrateur. Toutefois, il ne faut pas se complaire dans la description et la recension; des études empiriques demeurent nécessaires pour faire avancer les connaissances en TC et être en mesure de bien guider les organisations et individus aux prises avec des enjeux de TC.

Un deuxième constat réside en l'absence de validation de modèle intégrateur. Ce constat n'est pas nouveau et a fait l'objet de plusieurs écrits antérieurs (par ex., Chagnon et al., 2009; Dagenais et Robert, 2012; Nutley, Walter et Davies, 2007). Dans la même veine, nos résultats suggèrent qu'il existe encore trop peu d'études permettant de confirmer de manière certaine l'efficacité des stratégies, des approches ou des modèles évoqués. Sur le plan pratique, ces lacunes font en sorte que les milieux ne savent trop quelle direction emprunter pour améliorer leurs stratégies de TC.

Dans le domaine de l'éducation, nous avons recensé une avancée à ce niveau, avec les travaux de Ramdé (2012). En effet, la thèse doctorale de l'auteur révèle un modèle de mesure d'utilisation des CIR. Le modèle, conçu pour les enseignants, comporte des 
facteurs qui évaluent le degré d'utilisation des CIR. Bien que le modèle exige d'être validé par d'autres études, il pourrait aider à l'élaboration future d'un premier modèle de TC en éducation.

L'analyse des documents recensés met en lumière le peu de connaissances approfondies disponibles portant sur les déterminants du niveau mésosystémique (par exemple, les capacités structurelles et organisationnelles de développement de soutien du TC). De plus, il semble qu'il soit pour l'instant impossible d'identifier si une variable ou condition joue un rôle plus déterminant que d'autres dans le processus de TC; ou encore si certaines variables sont plus importantes que d'autres selon le contexte d'implantation. Étant donné que le succès du TC est multifactoriel, une connaissance plus fine de ses "meilleurs" déterminants en fonction du contexte s'avère souhaitable.

Les déterminants individuels du TC, tels que la motivation des utilisateurs des CIR, leurs traits de personnalité ou leurs compétences, demeurent à investiguer. Bien que des études se soient penchées sur la question (par ex., Mehiriz et Marceau, 2012; Osterling et Austen, 2008), les connaissances restent limitées. Par exemple, qui sont les utilisateurs de CIR? Peut-on les distinguer des "non-utilisateurs"? Dans le domaine de l'éducation, entre autres, il est étonnant qu'aucune étude n'ait examiné le rôle de la formation initiale sur l'utilisation des CIR. En effet, la formation initiale des enseignants permet à ces derniers d'acquérir des stratégies et des compétences d'enseignement - activité qui vise entre autres à transmettre des connaissances aux élèves. Toutefois, il est essentiel que la formation initiale permette également aux enseignants de devenir de meilleurs utilisateurs des CIR, tout au long de leur carrière. Ainsi, on peut penser qu'une meilleure formation en méthodologie de recherche serait profitable pour développer les compétences nécessaires à une bonne utilisation des CIR.

Plus précisément, les professeurs œuvrant en formation initiale pourraient outiller les étudiants en enseignement sur les aspects suivants: la recherche d'articles scientifiques, la compréhension des critères de scientificité, la distinction des types de recherche, la valeur des différentes publications, l'interprétation des résultats, etc. Il pourrait aussi être question dans les cours de supervision de stage ou les formations qui s'y prêtent d'inviter les étudiants à produire de courts travaux de recherche en lien avec des problématiques vécues lors des stages. La mise en place de sous-groupes de discussion sur les thèmes documentés par les étudiants pourrait s'ajouter en complément d'activité. L'idée étant de développer tant leur autonomie que des comportements sociaux orientés sur le TC. 
Les résultats de notre étude incitent également à aborder ces contenus méthodologiques avec nuance, en prenant en compte les champs d'intérêt des étudiants et le stade où ils sont. En effet, selon Crahay et al. (2010), les étudiants en enseignement seraient surtout interpelés par l'aspect relationnel et la gestion de classe. Ces contenus récolteraient donc une meilleure réceptivité de leur part, ce qui aurait avantage à être considéré en formation initiale. S'il faut ouvrir les horizons des étudiants avec des thèmes variés, la prise en compte de ce qui les interpelle plus particulièrement est à considérer.

En lien avec le point précédent, il parait tout aussi nécessaire de reconnaitre le rôle et l'influence des croyances (enracinées dans les connaissances tacites) dans la résistance au TC chez les acteurs scolaires. L'instauration de mesures d'accompagnement et d'un suivi s'inscrivant dans le long terme en formation continue pourrait se révéler utile à cet effet, cela, tant pour externaliser ces savoirs d'expériences (dont certains ont leur pertinence) que pour soutenir les acteurs dans leur processus de TC. En outre, une sensibilisation à cette réalité pourrait être effectuée en formation initiale.

Enfin, nos résultats révèlent que les principaux enjeux du TC identifiés avant 2007 demeurent présents tant dans la pratique que dans la recherche, et ce, malgré les avancées réalisées depuis. Qui plus est, tous les domaines sont touchés et non pas seulement l'éducation.

\section{Conclusion}

Cet article visait à rendre compte de l'évolution des connaissances produites sur le transfert des CIR en éducation selon un regard croisé avec la situation dans l'ensemble des domaines. Le texte fait ainsi état du rôle majeur des organisations, mais aussi de l'importance que soient pris en compte les utilisateurs dans tout processus de TC, incluant leur formation initiale. II rend compte également de lacunes concernant le TC: absence de taxonomie commune, de modèle intégrateur, de moyens d'évaluation efficaces, méconnaissance des meilleurs déterminants ou combinaisons de variables (organisationnelles et individuelles) susceptibles de maximiser le processus. Enfin, cet état des lieux permet de constater que les enjeux du TC ne sont pas circonscrits à quelques domaines; tous les domaines sont touchés, révélant ainsi le caractère transversal et multidisciplinaire du transfert des connaissances. 


\section{Références}

Abdoulaye, A. (2003). Conceptualisation et dissémination des "bonnes pratiques" en éducation: essai d'une approche internationale à partir d'enseignements tirés d'un projet. Activité de démonstration en éducation pour le développement durable. Document téléaccessible à l'adresse <http://www.unesco.org/education/desd>.

Abrami, P.C., Lysenko, L., Janosz, M., Bernard, R.M. et Dagenais, C. (2010). L'utilisation des connaissances issues de la recherche par les écoles. In M. Janosz, J. Bélanger, C. Dagenais, F. Bowen et P.C. Abrami (dir.), Rapport final d'évaluation de la stratégie d'intervention Agir autrement (S3/ p. 1-51). Groupe de recherche sur les environnements scolaires, Université de Montréal.

Albaladejo, C., Geslin, P., Magda, D. et Salembier, P. (2009). La mise à l'épreuve: le transfert des connaissances scientifiques en questions. Paris: Éditions Quae.

Arksey, H. et O'Malley, L. (2005). Scoping studies: Towards a methodological framework. International Journal of Social Research Methodology: Theory \& Practice, 8, 19-32.

Ash, J., Sittig, D., Guappone, K., Dykstra, R.H., Richardson, J., Wright, A. et al. (2012). Recommended practices for computerized clinical decision support and knowledge management in community settings: A qualitative study. BMC Medical Informatics and Decision Making, 12(1), 6.

Bammer, G., Michaux, A. et Sanson, A. (dir.) (2010). Bridging the "Know-do" Gap: Knowledge Brokering to Improve Child Wellbeing. Canberra, Australie: ANU E Press.

Becheikh N., Ziam S., Idrissi O., Castonguay, Y. et Landry, R. (2010). How to improve knowledge transfer strategies and practices in education? Answers from a systematic literature review. Research in Higher Education Journal, 7, 1-21.

Belleau, H. (2011), De la mobilisation des connaissances au partenariat de recherche: le cas du "Portrait des jeunes du quartier Bordeaux-Cartierville» à Montréal. SociologieS. Document téléaccessible à l’adresse <http://sociologies.revues.org/3730>.

Besner, F., Laurendeau, M.-C. et Bergeron, P. (2012). La formation en partage et utilisation des connaissances sous l'angle de l'apprenant. Canadian Journal of Public Health, 103(3), 235-237.

Biasutti, M. et El-Deghaidy, H. (2012). Using Wiki in teacher education: Impact on knowledge management processes and student satisfaction. Computers \& Education, 59, 861-872.

Biesta, G. (2007). Why "what works" won't work: Evidence-based practice and the democratic deficit in educational research. Educational Theory, 57(1), 1-22.

Bourdeau, A. (2011). Élaboration de profils de compétences et d'outils d'évaluation pour les postes de la Direction du développement des personnes et de l'organisation de Services Québec et propositions concernant les besoins de formation, ainsi que la mise en place de stratégies de transfert des connaissances. Rapport de stage en vue de l'obtention de la maîtrise, ENAP, Québec. 
Bowen, S., Martens, P. et The Manitoba Need to Know Team (2005). Demystifying knowledge translation: Learning from the community. Journal of Health Services Research and Policy, 10(4), 203-211.

Bricout, J.C., Pollio, D.E., Edmond, T. et McBride, A.M. (2008). Macro practice teaching and curriculum development from an evidence-based perspective. Journal of EvidenceBased Social Work, 5(3), 597-621.

Brodeur, M., Deaudelin, C. et Bru, M. (2005). Le développement professionnel des enseignants: apprendre à enseigner pour soutenir l'apprentissage des élèves. Revue des sciences de l'éducation, 31(1), 5-14.

Brodeur M., Fontan J-M., Landry R., Auclair M. et Tirilly, M. (2009). L'UQAM: une mission particulière de mobilisation des connaissances. Rapport présenté au Vice-recteur à la recherche et à la création, Université du Québec à Montréal.

Broekkamp, H. et Van Hout-Wolters, B. (2007). The gap between educational research and practice: A literature review, symposium, and questionnaire. Educational Research and Evaluation: An International Journal on Theory and Practice, 13(3), 203-220.

Brown, C. (2012). Adoption by policy makers of knowledge from educational research: An alternative perspective. Issues in Educational Research, 22(2), 91-110.

Butler, H., Novak Lauscher, S., Jarvis-Selinger, B. et Beckingham, B. (2004). Collaboration and self-regulation in teachers' professional development. Teaching and Teacher Education, 20, 435-455.

Campbell, C. (2011). Mobilisation des connaissances en éducation. Colloque ontarien sur la recherche en éducation. Ministère de l'Éducation de l'Ontario.

Chabot, A. (2007). Évaluation qualitative des déterminants de l'utilisation des connaissances issues de la recherche par les enseignants d'écoles secondaires québécoises en milieu défavorisé. Mémoire de recherche inédit, Université de Montréal.

Chagnon, F., Daigle, M., Gervais, M.-J., Houle, J. et Béguet, V. (2009). Le transfert de l'évaluation fondée sur la théorie du programme comme stratégie d'application des connaissances issues de la recherche. The Canadian Journal of Program Evaluation, 23(1), 3-32.

Chagnon, F. et Gervais, M.-J. (2011). Modélisation des déterminants et des retombées de l'application des connaissances issues de la recherche psychosociale: partie II étude de validation. Québec: Fonds québécois de recherche sur la société et culture.

Cloutier, Y. (2010). Mobilisation et transfert de connaissances dans le cadre d'une expérience de concertation. Essai de recherche inédit, Institut national de la recherche scientifique, Université du Québec.

Collerette, P. (2008). Pour une gestion du changement discipline dans l'administration publique. Télescope, 14(3), 33-49. 
Colluci-Cray, L. et Fraser, C. (2008). Contested aspects of becoming a teacher: Teacher learning and role of subject knowledge. European Educational Research Journal, 7, 475-486.

Cooper, A. (2013). Knowledge mobilisation in education across Canada: A cross-case analysis of 44 research brokering organisations. Evidence and Policy, 31, 29-59.

Cooper, A. et Levin, B. (2013). Research use by leaders in Canadian school districts. International Journal of Education Policy and Leadership, 8(7), 1-15.

Cordingley, P. (2008). Research and evidence-informed practice: Focusing on practice and practitioners. Cambridge Journal of Education, 38(1), 37-52.

Crahay, M., Wanlin, P., Issaieva, É. et Laduron, I. (2010). Fonctions, structuration et évolution des croyances (et connaissances) des enseignants. Revue française de pédagogie, 172, 85-129.

Crona, B.I. et Parker, J.N. (2011). Network determinants of knowledge utilization: Preliminary lessons from a boundary organization. Science Communication, 33(4), 448-471.

Dagenais, C. (2012). Émergence de la première équipe de recherche en partenariat sur le transfert des connaissances dans le domaine social. In C. Dagenais et É. Robert, (dir.), Le transfert des connaissances dans le domaine social (p. 13-33). Montréal : Les Presses de l’Université de Montréal.

Dagenais, C. et Robert, É. (2012). Introduction. In Dagenais, C. et Robert, É. (dir.), Le transfert des connaissances dans le domaine social (p. 7-12). Montréal : Les Presses de l'Université de Montréal.

Dagenais, C., Lysenko, L., Abramai, P.C., Bernard, R.M., Ramdé, J. et Janosz, M. (2012). Use of research-based information by school practitioners and determinants of use: A review of empirical research. Evidence \& Policy, 8(3), 285-309.

Dagenais, C., Janosz, M. et Dutil, J. (2008). Étude des besoins des chercheurs de I'Université de Montréal en matière de transfert des connaissances issues de la recherche. Rapport présenté au Bureau de la Recherche-Développement-Valorisation de I'Université de Montréal, Québec.

Dagenais, C., Janosz, M., Abrami, P. et Bernard, R. (2007). Examen des mécanismes en jeu dans la décision des intervenants scolaires d'utiliser les connaissances issues de la recherche pour changer leurs pratiques. Rapport présenté au Programme de recherche sur la persévérance et la réussite scolaire, Université de Montréal.

Davidson, K. et Nowicki, E. (2012). An exploration of the utility of a knowledge utilization framework to study the gap between reading disabilities research and practice. Alberta Journal of Educational Research, 58(3), 330-349.

Davies, H.T.O. et Nutley, S.M. (2008). Learning More About How Research-Based Knowledge Gets Used: Guidance in the Development of New Empirical Research. William T. Grant Foundation, New York. 
Deauvieau, J. (2005). Devenir enseignant du secondaire: les logiques d'accès au métier. Revue française de pédagogie, 150, 31-41.

Deslandes, R. et Bertrand, R. (2004). Motivation des parents à participer au suivi scolaire de leur enfant au primaire. Revue des sciences de l'éducation, 30(2), 411-433.

Dionne, L. (2003). La collaboration entre collègues comme mode de développement professionnel chez l'enseignant: une étude de cas. Thèse de doctorat inédite, Université du Québec à Montréal.

Elissalde, J. et Renaud, L. (2010). Les démarches de circulation des connaissances: mobilisation et valorisation des connaissances. In L. Renaud (dir.), Les médias et la santé: de l'émergence à l'appropriation des normes sociales (p. 409-429). Québec: Presses de l'Université du Québec.

Elissalde, J., Gaudet, J. et Renaud, L. (2010). Circulation des connaissances: modèle et stratégies. Revue internationale Communication sociale et publique, 3(4), 135-149.

Edelstein, H. (2011). Les pratiques collaboratives d'organismes publics utilisant Internet pour la mobilisation du savoir dans le domaine de l'éducation. Télescope, 17(3), 140-157.

Flessner, R. (2012). Addressing the research/practice divide in teacher education. Action in Teacher Education, 34(2), 159-171.

Florin, J., Ehrenberg, A., Wallin, L. et Gustavsson, P. (2011). Educational support for research utilization and capability beliefs regarding evidence-based practice skills: A national survey of senior nursing students. Journal of Advanced Nursing, 68(4), 888-897.

Fonds de recherche québécois sur la société et la culture (FRQSC) (2011). Plan d'action en matière de transfert de connaissances 2011-2014. Gouvernement du Québec. Document téléaccessible à l'adresse <http://www.fqrsc.gouv.qc.ca/upload/ publications-fqrsc/fichiers/publication_52.pdf $>$.

Fournier, M. (2011). Éduquer et former: connaissances et débats en éducation et formation. France: Éditions Sciences Humaines.

Gagnon, K. (2012). Le transfert de connaissances dans l'insertion professionnelle des enseignants au collégial. Education Canada. Document téléaccessible à l'adresse <http://www.cea-ace.ca/education-canada/article>.

Gera, R. (2012). Bridging the gap in knowledge transfer between academia and practitioners. International Journal of Educational Management, 26(3), 252-273.

Gervais, M-J., Marion, C., Dagenais, C., Chiocchio, F. et Houlfort, N. (2015). Dealing with the complexity of evaluating knowledge transfer strategies: proposed guiding principles for developing valid instruments. Research Evaluation, 34. Document téléaccessible à l'adresse <http://doi.org/10.1093/reseval/rvv034>. 
Gervais, M.J., Chagnon, F. et Paccioni, A. (2011). Augmenter l'utilisation des données probantes par les intervenants et les gestionnaires en centre jeunesse. Service social, $57(1), 49-62$.

Gervais, M.J. et Chagnon, F. (2010). Modélisation des déterminants et des retombées de l'utilisation des connaissances issues de la recherche psychosociale. Chaire d'étude sur l'application des connaissances dans le domaine des jeunes et des familles en difficulté, Université du Québec à Montréal.

Geuna, A. et Muscio, A. (2008). The governance of university knowledge transfer. Science and Technology Policy Research, 173, 1-30.

Gurtner, J-L., Gulfi, A., Monnard, I. et Schumacher, J. (2005). Est-il possible de prédire l'évolution de la motivation pour le travail scolaire de l'enfance à l'adolescence? Revue française de pédagogie, 155, 21-33.

Harvey, J.-F. (2011). Comment favoriser le partage des connaissances? Le cas des communautés de pratique pilotées. Gestion, 35(4), 73-80.

Hedges, H. (2010). Blurring the boundaries: Connecting research, practice and professional learning. Cambridge Journal of Education, 40(3), 299-314.

Hemsley-Brown, J. et Sharp, C. (2003). The use of research to improve professional practice: A systematic review of the literature. Oxford Review of Education, 29(4), 449-470.

Hemsley-Brown, J. (2004). Facilitating research utilisation: A cross-sector review of research evidence. The International Journal of Public Sector Management, 17(6/7), 534-552.

Hofstetter, R. et Schneuwly, B. (2009), Savoirs en (trans)formation: au cour des professions de l'enseignement et de la formation. Bruxelles: Éditions De Boeck Université.

Hotho, J.J., Becker-Ritterspach, F. et Saka-Helmhout, A. (2012). Enriching absorptive capacity throught social interaction. British Journal of Management, 23, 383-401.

Jbilou, J. (2010). Adaptation des résultats de recherche. Thèse de recherche inédite, Université Laval, Québec.

Kirst, M.W. (2000). Bridging education research and education policymaking. Oxford Review of Education, 26, 379-391.

Lacroix, S. (2012). Transfert et mobilisation des connaissances dans le cadre d'une recherche sur la conciliation travail-famille dans les organismes culturels au Québec. Essai de recherche inédit, Institut national de la recherche scientifique, Université du Québec.

Lamari, M. (2012). Le courtage de connaissances à l'ère du numérique: portrait empirique des pratiques émergentes et examen de leurs impacts dans le secteur de la santé au Canada. Les Cahiers du numérique, 1(8), 97-130. 
Lamari, M. (2010). Le transfert intergénérationnel des connaissances tacites: les concepts utilisés et les évidences empiriques démontrées. Télescopes, 16, 39-65.

Landry, R. (2010). Mobiliser la recherche pour la réussite scolaire: stratégie et pistes d'action favorisant le transfert des données de recherche dans les pratiques pédagogiques, Communication présentée le 10 juin 2010, Journée d'étude sur la mobilisation des connaissances issues de la recherche en éducation, Université du Québec à Montréal.

Landry, R., Becheikh, N., Amara, N., Ziam, S., Idrissi, O. et Castonguay, Y. (2008). La recherche, comment s'y retrouver? Revue systématique des écrits sur le transfert des connaissances en éducation. Ministère de l'Éducation, Gouvernement du Québec.

Lavis, J., Robertson, D., Woodside, J., McLeod, C. et Abelson, J. (2003). How can research organizations more effectively transfer research knowledge to decision-makers? The Milbank Quarterly, 81(2), 221-248.

Lemire, J., Bigras, N. et Eryasa, J. (2011). L'échange de connaissances en petite enfance: état des lieux. In N. Bigras et C. Bouchard (dir.), L'échange de connaissances en petite enfance: comment mettre à profit les expertises des chercheurs et des praticiens (p. 9-31). Québec: Les Presses de l'Université du Québec.

Lemire, N., Souffez, K. et Laurendeau, M.C. (2009). Animer un processus de transfert des connaissances: bilan des connaissances et outil d'animation. Direction de la recherche, formation et développement, Institut national de santé publique du Québec.

Lenoir, Y. et Vanhulle, S. (2006). Étudier la pratique enseignante dans sa complexité: une exigence pour la recherche et la formation à l'enseignement. In A. Hasni, Y. Lenoir et J. Lebeaume (dir.), La formation à l'enseignement des sciences et des technologies au secondaire dans le contexte des réformes par compétences (p.193-245). Québec: Presses de l'Université du Québec.

Levac, D., Colquhoun, H. et O'Brien, K. (2010). Scoping studies: Advancing the methodology. Implementation Science, 5, 1-9.

Levin, B. (2010) Leadership for evidence-informed education. School Leadership \& Management: Formerly School Organisation, 30(4), 303-315.

Levin, B., Cooper, A., Arjomand, S. et Thompson, K. (2011). L'utilisation de la recherche et son incidence dans les écoles secondaires: une étude examinant la mobilisation des connaissances en éducation. Association canadienne d'éducation (ACE).

Lessard, C., Altet, M., Paquay, L. et Perrenoud, P. (2004). Entre sens commun et sciences humaines. Quels savoirs pour enseigner?, Bruxelles: De Boeck.

Lin, C. et Chen, M. (2009). Factors affecting teachers' knowledge sharing behaviors and motivation: System functions that work. eLAC, 1-8. 
Long, J.C., Cunningham, F.C. et Braithwaite, J. (2013). Bridges, brokers and boundary spanners in collaborative networks: A systematic review. BMC Health Services Research, 13(158), 1-13. Document téléaccessible à l'adresse <http://link.springer. com/article/10.1186\%2F1472-6963-13-15>.

Martin, M.-L., Jensen, E., Coatsworth-Puspoky, R., Forchuk, C., Lysiak-Globe, T. et Beal, G. (2007). Integrating an evidenced-based research intervention in the discharge of mental health clients. Archives of Psychiatric Nursing, 21(2), 101-111.

Maubant, P. et Roger, L. (2012). Les métiers de l'éducation et de la formation: une professionnalisation en tensions. Revue internationale de pédagogie de l'enseignement supérieur, 28(1), 1-14.

McCloskey, D. (2008). Nurses'perceptions of research utilization in a corporate health care system. Journal of Nursing Scholarship, 40, 39-45.

Meagher, L. (2013). The invisible made visible: Using impact evaluations to illuminate and inform the role of knowledge intermediaries. Evidence \& Policy, 9(3), 409-418.

Mehiriz, K. et Marceau, R. (2012). Les déterminants de l'utilisation des recherches en éducation: le cas des conseillers pédagogiques au Québec. McGill Journal of Education, 47(1), 231-250.

Meyer, M. (2010). The Rise of the Knowledge Broker. Science Communication, 32(1), 118-127.

Nelson, S.R., Leffler, J.C. et Hansen, B.A. (2009). Toward a Research Agenda for Understanding and Improving the Use of Research Evidence. Portland, OR: Northwest Regional Educational Laboratory. Document téléaccessible à l'adresse <www.nwrel. org/researchuse/report.pdf $>$.

Nutley, S., Walter, I. et Davies, H.T.O. (2007). Using Evidence: How Research can Inform Public Services. Bristol: The Policy Press.

Ouimet, M., Bédard, P-O. et Lapointe, L. (2012). De la nature de la connaissance scientifique et de quelques enjeux liés à son transfert et à son utilisation. In C. Dagenais et É. Robert (dir.), Le transfert des connaissances dans le domaine social, (p. 35-43). Montréal: Les Presses de l'Université de Montréal.

Osterling, K.L. et Austin, M. (2008). The dissemination and utilization of research for promoting evidence-based practice. Journal of Evidence-Based Social Work, 5(1-2), 295-319.

Paroutis, S. et Saleh, A.A. (2009). Determinants of knowledge sharing using Web 2.0 technologies. Journal of Knowledge Management, 13(4), 52-63.

Perrenoud, P. (2008). Dix nouvelles compétences pour enseigner: invitation au voyage. Paris: ESF Éditeur. 
Perrenoud, P., Altet, M., Lessard, C. et Paquay, L. (2008). Conflit de savoirs en formation des enseignants. Entre savoirs issus de la recherche et savoirs issus de l'expérience. Bruxelles: De Boeck Université.

Presse, M-C. (2009). Analyse des pratiques et transformations des pratiques: une question de valeurs? In C. Albaladejo, P. Geslin, D. Magda et P. Salembier (dir), La mise à l'épreuve: le transfert des connaissances scientifiques en questions (p. 141-156). Paris: Éditions Quae.

Ramdé, J. (2012). Utilisation des connaissances issues de la recherche en éducation: validation d'un questionnaire et proposition d'un modèle. Thèse de doctorat inédite, Université de Montréal.

Ridde, V. (2009). Le transfert de connaissances et les règles de fonctionnement du système universitaire: besoin de changements. Global Health Promotion, 16(3), 70-72. Ridde, V., Dagenais, C. et Boileau, M. (2013). Une synthèse exploratoire du courtage en connaissance en santé publique. Santé publique, 2(25), 137-145.

Rioux A.F. et Chevrollier, J.P. (2010) Devenir enseignant: quels "Choix" inconscients? Étude de cas. Annales Médico-Psychologiques, 167(5), 370-384.

Sa, C.M., Li, S.X. et Faubert, B. (2010). Faculties of education and institutional strategies for knowledge mobilization: An exploratory study. High Educ, 61, 501-512.

Saussez, F. et Lessard, C. (2009). Entre orthodoxie et pluralisme, les enjeux de l'éducation basée sur la preuve. Revue française de pédagogie, 168, 111-136.

Saussez F. et Paquay L. (2004). Tirer profit de la tension entre "concepts quotidiens" et "concepts scientifiques". Quels espaces de formation et de recherche construire? In C. Lessard, M. Altet, L. Paquay et P. Perrenoud (dir.), Entre sens commun et sciences humaines. Quels savoirs pour enseigner? (p. 115-138). Bruxelles: De Boeck.

Shonkoff, J.P. et Bales, S.N. (2011). Science does not speak for itself: Translating child development research for the public and its policymakers. Child Development, 82(1), 17-32.

Souffez, K. (2012). État des connaissances dur le transfert de connaissances dans le domaine de la santé. In C. Dagenais et É. Robert (dir.), Le transfert des connaissances dans le domaine social (p. 35-43). Montréal: Les Presses de l’Université de Montréal.

Spencer, T.D., Detrich, R. et Slocum, T.A. (2012). Evidence-based Practice: A Framework for Making Effective Decisions. Education and Treatment of Children, 35(2), 127-151.

St-Cyr Tribble, D., Lane, J., Boyer, G., Aubé, D., Blackburn, F., Brassard, C., Gendron, S., Labadie, J-F., Belleau, H. et Le Gall, J. (2008). Le cadre de référence "trans-action" en transfert des connaissances. Rapport de recherche: le transfert des connaissances entre praticiens, gestionnaires et chercheurs comme source d'innovation en CLSC, Direction de la coordination et des affaires académiques, Université de Sherbrooke. 
Sylva, K., Taggart, B., Melhuish, E., Sammons, P. et Siraj-Blatchford, I. (2007). Changing models of research to inform educational policy. Research Papers in Education, 22(2), 155-168.

Thibodeau, S., Dussault, M., Frenette, E. et Royer, N. (2011). Solitude professionnelle d'enseignants du secondaire: relations avec le leadership du directeur d'école et leurs croyances d'autoefficacité sociale. Revue canadienne de l'éducation, 34(4), 177-199.

Trevithick-Oglesby, D. (2008). Using A Special Education Dialogue Guide For Knowledge Transfer. Thèse, George Washington University.

Tseng, V. (2012). The uses of research in policy and practice. Social Policy Report. Society for Research in Child Development, 26(2), 2-24.

Veillard, L. et Coppé, S. (2009). Mobilisation de connaissances antérieures en formation professionnelle par alternance. Éducation et didactique, 3(2), 47-76.

Ward, V., House, A. et Hamer, S. (2009). Knowledge brokering: Exploring the process of transferring knowledge into action. BMC Health Services Research, 9(12), 1-6.

Winton, P.J. et McCollum, J.A. (2008). Preparing and supporting high-quality early childhood practitioners: Issues and evidence. In P. Winton, J. McCollum, et C. Catlett (dir.), Practical approaches to early childhood professional development: Evidence, strategies, and resources (p. 1-12). Washington, DC: Zero to Three.

Ziam, S. (2010). Les déterminants de la capacité d'absorption des connaissances: le cas des courtiers de connaissances ceuvrant dans le domaine de la santé au Canada. Thèse de doctorat inédite, Université Laval. 